\title{
Magnetic Helicity Generated together with Evolution of the Large-Scale Magnetic Field
}

\author{
Pavel Ambrož \\ Astronomical Institute of the Acad. Sci. of the Czech Rep., CZ-25165 \\ Ondrejov , Czech Republic
}

Abstract. Text below is an extended abstract of the poster, presented on JD03 session during 25th GA IAU in Sydney.

Large-scale magnetic regions evolve their structure in the photosphere relatively slowly during their many months long lifetime. Their evolution is partly influenced by turbulent diffusion, but also by large-scale velocity fields with both axially symmetric and non-axially symmetric components. In the latter case, horizontal velocities vary in both longitude and latitude. The horizontal transport velocities responsible for the evolution of the magnetic flux were inferred for each Carrington rotation (CR) for each of the last three activity cycles Nos. 21, 22 and 23. The measurements of magnetic field from WSO of Stanford University were used. Zonal transport velocities averaged over longitude during each CR give a very good approximation of the latitude-dependent differential rotation. The velocity distribution, however, demonstrates that non-axially symmetric horizontal flows create large-scale eddies and helical structures. They are persistent, and the structures persist for up to $4 \mathrm{CRs}$. The detected flow systems create discrete giant flow patterns that are from 40 to 60 heliographic degrees wide in the equatorial zone. From the surface distribution of horizontal velocities we computed for each CR more than 2500 values of relative "shear" velocities $\Delta \mathbf{v}$ and we determined regions where due to shear the maximal helical turbulence can occur. Such regions are necessary for generation of magnetic helicity, but another necessary condition is the value of the total magnetic field $\mathbf{B}$. Total values were summed from measured $\mathbf{B}_{\mathbf{m}, \mathbf{r}}$ (radial) and current-free extrapolated $\mathbf{B}_{\mathbf{p}, \mathbf{t}}$ (transversal) components. Product of $\mathbf{B}^{\mathbf{2}}$ and $\Delta \mathbf{v}$ can be expressed in the form relating with the "available" magnetic energy. This is considered to be a part of the magnetic energy, which can be released when is above the threshold of instability of the coronal magnetic configuration. Regions with high rate of available energy were tested with about 95 thousand specific values of flare index derived from observed $H_{\alpha}$ flares. The occurrence of flares was strictly concentrated in regions with a high rate of available energy. The "shear" regions are frequently distributed in the solar photosphere and usually don't coincide with the "shear zone" expected due to axially symmetric differential rotation. Such a zone is nearly absent in our results. The "shear" regions frequently coincide with the strong magnetic fields of the active or bipolar magnetic regions, and therefore, during each $\mathrm{CR}$, only a very limited number of regions are able to produce and transport a concentrated amount of magnetic helicity to the corona.

Study was supported by GAČR 205/10/0657 and GA AVČR S1003006. 